\title{
Acute Disseminated Melioidosis Presenting with Septic Arthritis and Diffuse Pulmonary Consolidation in an Otherwise Healthy Adult: A Case Report
}

Hai Sherng Lee, ${ }^{1}$ Abdul Azeez Ahamed Riyaaz, ${ }^{1}$ Seng Hong Yeoh. ${ }^{1}$

\begin{abstract}
Background: Melioidosis is an infectious disease caused by Burkholderia pseudomallei. It is most prevalent in South-East Asia, northern Australia, and the Indian subcontinent. Septic arthritis is a rare manifestation of melioidosis. Melioidosis is usually found in patients with diabetes, heavy alcohol use, or chronic lung disease. Case: We report a case of melioidosis in an otherwise healthy 44-year-old male, who presented with acute painful left knee swelling, high-grade fever associated with chills, rigors and night sweats, and a productive cough. Examination revealed active synovitis with effusion involving his left knee, ankle and elbow joints and scattered crackles over both lung fields. Chest X-ray showed diffuse pulmonary consolidation. Abdominal ultrasound showed splenic micro-abscesses. The diagnosis was made based on a positive blood culture for Burkholderia pseudomallei. He was started on appropriate antibiotics and responded well, becoming afebrile after 48 hours, while his joint effusions disappeared after one week. Conclusion: Septic arthritis only occurs in $4 \%$ of patients with melioidosis. When there is diffuse pulmonary involvement, melioidosis may mimic disseminated tuberculosis, sepsis syndromes, and systemic vasculitis syndromes. This case is relevant for medical literature as melioidosis is emerging and is expanding its territories worldwide. It should be considered early in the differential diagnoses in endemic areas so that treatment can be started early to reduce its high mortality and morbidity.
\end{abstract}

Keywords: Abscess, Adult, Arthritis Infectious, Burkholderia pseudomallei, Melioidosis (Source: MeSH, NLM).

\section{Introduction}

About the Author: $\mathrm{Dr} \mathrm{Ha}$ Sherng Lee is currently working at the Wollongong Hospital in New South Wales, Australia. He graduated from Monash University and was awarded, in his final year, "Excellence in Pre-Intern Assessment" under the Acute General Surgery Unit at the School of Clinical Sciences, Monash Health, Victoria, Australia.
Melioidosis is an infectious disease caused by the gram-negative saprophyte Burkholderia pseudomallei. It is most prevalent in South-East Asia, northern Australia, and the Indian subcontinent. ${ }^{1}$ It is usually found in patients with diabetes, heavy alcohol use, or chronic lung disease. ' The common manifestations are pneumonia (in half of all cases), genitourinary infection, skin infection, and bacteraemia without evident focus. ${ }^{2}$ Complications include septic shock. Recurrent melioidosis is common unless long courses of treatment are given, and high mortality rates ranging from $14 \%$ to $40 \%$ have been reported despite optimal therapy. ${ }^{1,2}$

This case is unusual as the patient had none of the risk factors known to be associated with this infection and presented with septic arthritis, which is a rare presenting feature. It is an uncommon but important disease for clinicians to consider as its manifestations are protean, and it can mimic many diseases, including pulmonary tuberculosis. ${ }^{1,2}$ Melioidosis should be considered as part of the differential diagnosis of patients with sepsis or abscesses in endemic regions. It is being recognized more frequently in these regions and is one of the important emerging infections that clinicians will encounter more in the future. ${ }^{2,3}$ Informed written consent was obtained from the patient for writing and publishing this case report.

Key Points:
- Melioidosis is endemic in tropical regions like South-East Asia and
northern Australia and is usually found in patients with diabetes, heavy
alcohol use, or chronic lung disease.
- Septic arthritis is a rare manifestation of melioidosis, found in only $4 \%$
of the cases at presentation.
- Mortality rates for melioidosis vary from $14 \%$ in Australia to $40 \%$ in
northeast Thailand.
- The standard treatment for melioidosis comprises $10-14$ days of intra-
venous ceftazidime or meropenem, followed by oral trimethoprim-sul-
famethoxazole (TMP-SMX) plus doxycycline taken every 12 hours for 3
to 6 months.
- Melioidosis is becoming an emerging infection and expanding its te-
rritories worldwide. Due to its high mortality and morbidity, it should
be considered early in the differential diagnoses of patients presenting
with constitutional symptoms in endemic areas.

\section{The Case}

A 44-year-old male of Chinese ethnicity, who was previously healthy, presented to a district hospital in the state of Johor, Malaysia on July 28, 2014, complaining of a one-week history of acute onset painful swelling of his left knee. He had also developed a productive cough with scanty yellowish sputum, as well as high-grade fever associated with chills, rigors, and night sweats of similar duration. Three days before hospital admission, his left ankle and elbow joints were noted to be swollen and painful. He denied any recent trauma. Findings from a review of his cardiovascular, gastrointestinal, and genitourinary systems were normal. His past 
medical history was unremarkable, and there was no family history of autoimmune diseases. He denied any recent travel outside his state and contact with people with active pulmonary tuberculosis. He was a current smoker and had smoked around 16 cigarette pack-years since the age of 18 . He drank alcohol only during social events and denied illicit drug use and high-risk sexual behaviours. He has worked as a tile layer for many years, during which he had occasional contact with the soil.

Examination revealed a man of average build, who appeared ill and mildly dyspnoeic. Upon admission, his vital signs included a pulse rate of 110 beats/min, a blood pressure of $110 / 61 \mathrm{mmHg}$, and a respiratory rate of 24 breaths/min. His temperature was $38.5^{\circ} \mathrm{C}$, and there were no pallor, icterus, lymphadenopathy, or skin rash noted. Respiratory examination revealed only scattered bilateral crackles while a mild hepatosplenomegaly was felt upon abdominal examination. His left knee, ankle, and elbow joints showed signs of active synovitis with effusion.

Investigations showed a white cell leukocytosis ( $\left.22.2 \times 10^{9} / \mathrm{L}\right)$ with neutrophilia (86.4\%) and deranged liver function tests [alanine transaminase (ALT): $56.2 \mathrm{U} / \mathrm{L}$, aspartate aminotransferase (AST): $54.7 \mathrm{U} / \mathrm{L}$, and alkaline phosphatase (ALP): 447.2 $\mathrm{U} / \mathrm{L}$ ]. Other investigations, including urine dipstick and renal profile, were normal. Chest X-ray showed bilateral diffuse patchy opacities suggestive of consolidation (Figure 1 ). An ultrasound of his abdomen confirmed mild hepatosplenomegaly and multiple irregular small hypo-dense lesions scattered in the spleen parenchyma, likely to represent micro-abscesses. At this point, a working diagnosis of septicaemia of unknown focus was made, and he was started on intravenous co-amoxiclav to cover the broad spectrum. Sputum samples were sent for acid-fast bacilli to rule out active pulmonary tuberculosis and came back negative. A human immunodeficiency virus (HIV) screening test was negative. He underwent aspiration of his left knee joint effusion, and a drainage catheter was inserted (Figure 2). The joint fluid was yellowish and clear, with no organisms detected on Gram's stain and culture.

On day two of hospital admission, his blood culture grew Burkholderia pseudomallei, leading to the diagnosis of disseminated melioidosis. He was started on intravenous ceftazidime $2 \mathrm{~g}$ three times daily and two tablets of oral co-trimaxazole $960 \mathrm{mg}$ twice daily based on sensitivity results. He became afebrile after 48 hours, while his joint effusions disappeared over the first week of antibiotic treatment. The patient had to stay as an inpatient for two weeks for the intensive phase of intravenous antibiotic therapy, during which he did not develop any complications. A repeat chest $\mathrm{X}$-ray done after two weeks of intensive antibiotic therapy showed marked radiological improvement (Figure 3). He was discharged home after two weeks, with a plan of continuation of oral co-trimaxazole for another 20 weeks and outpatient follow-up at 4-6 weeks intervals. Our patient believed that he acquired the infection at his workplace and was satisfied with the treatment he received. He understood the protracted nature of his illness and was committed to completing his prolonged antibiotic therapy.
Figure 1. Patient's Chest X-ray, Showing Bilateral Diffuse Patchy Opacities Suggestive of Consolidation.

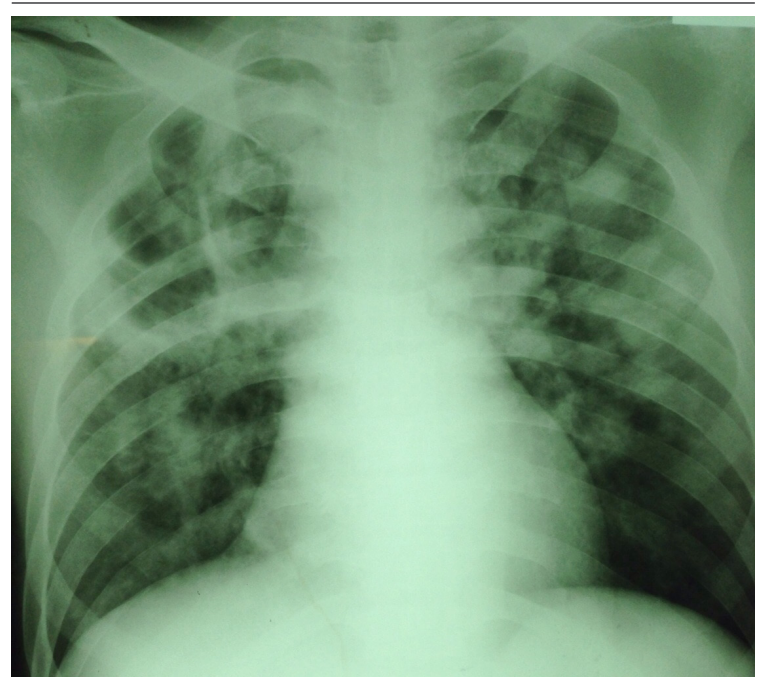

Figure 2. Patient's Left Knee with Effusion that had been Bandaged and Drained.

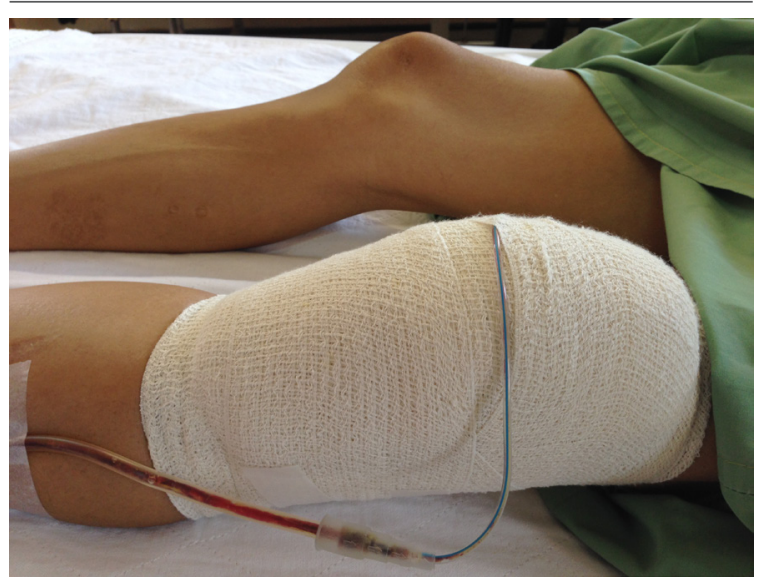

Figure 3. Patient's Repeat Chest X-ray After Two Weeks of Antibiotics, Showing Marked Radiological Improvement.

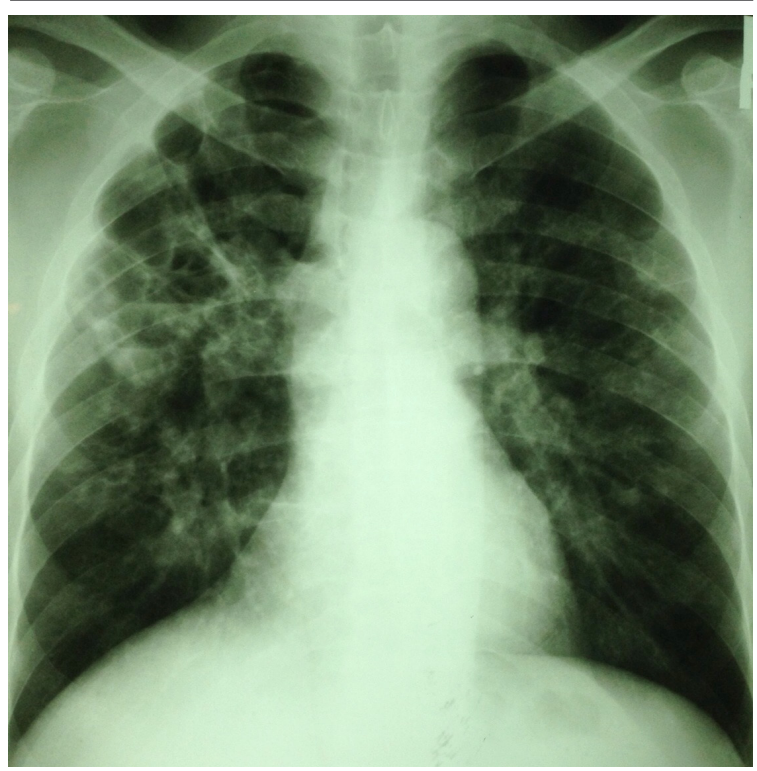




\section{Discussion}

Melioidosis is caused by the environmental Gram-negative soil saprophyte B. pseudomallei. Melioidosis is endemic in South-East Asia and northern Australia, with increasing recognition in the Indian subcontinent and elsewhere in the tropics. Northeast Thailand reports the highest number of cases, with an annual incidence of 50 cases per 100,000 people, making melioidosis the third most common cause of death due to infectious diseases in northeast Thailand.' In Darwin, tropical Australia, a 20-year prospective study from October 1989 yielded 540 cases of melioidosis. ${ }^{2}$ Melioidosis has been studied extensively only in certain regions of Malaysia where there is known to be a higher incidence of disease. For instance, there were 44 new cases of melioidosis in the Malaysian state of Johor between January 1999 and December 2003. ${ }^{4}$ Melioidosis is an emerging disease, as evidenced by increased reported incidence in endemic regions. For example, in Darwin, the prospective study identified 88 cases in the first five years, which increased steadily to 149 cases in the final five years. ${ }^{2}$ In Northeast Thailand, there were 198 cases in 1997, which increased to 380 cases in $2006 .^{3}$ In addition, cases are being identified in new areas where melioidosis has not previously been reported.

The primary modes of transmission of melioidosis are thought to be percutaneous inoculation in persons who are in regular contact with soil and water and, less commonly, via inhalation during severe stormy weather or by ingestion of contaminated food or water. 'Melioidosis is predominantly seasonal, and more than $75 \%$ of cases occur during the rainy season. $B$. pseudomallei can invade macrophages and survive and replicate for extended periods of time. Phagocytes may be able to destroy the bacteria, but some bacteria can escape endocytic vacuoles and enter into the cytoplasmic space. They are also capable of infecting other cells through actin-based membrane protrusions.

Melioidosis has a wide array of clinical signs and symptoms. It has an incubation period of 1-21 days, with a mean of 9 days, although prolonged periods of latency (up to 62 years) have been reported.' Its severity varies from an acute fulminant septic illness to a chronic infection, in which symptoms last for more than two months and may mimic malignancy or tuberculosis. In a descriptive study done over 20 years in tropical Australia, the most common presenting feature was pneumonia, which was present in $50 \%$ of cases, followed by genitourinary infection, skin infection, and bacteraemia without evident focus. $^{2}$ Septic arthritis, which was the principal presentation of our patient, and septic osteomyelitis are rare presenting features, with only $4 \%$ of cases having such manifestations. Over half of patients have bacteraemia on presentation, and septic shock develops in approximately one fifth. Internal-organ abscesses and secondary foci in the lungs, skin and soft tissues, bones and joints, or any other organ may occur. Up to $80 \%$ of patients with melioidosis have one or more risk factors for the disease, which include diabetes, heavy alcohol use, chronic pulmonary disease, chronic kidney disease, and thalassaemia. Glucocorticoid therapy and cancer were only associated in less than $5 \%$ of cases. ${ }^{1-3}$ Our patient, however, had no such known risk factors.

The diagnosis of melioidosis is made from a positive culture for B. pseudomallei from any clinical sample, which in our patient's case was a positive blood culture. We would have faced diagnostic difficulties if the culture had been negative due to the non-availability of serological or genetic-based testing at the hospital where the patient was treated. But the delay in the identification of $B$. pseudomallei or misidentification as another species is not uncommon in laboratories that are unfamiliar with this organism..$^{5}$ A direct polymerase-chain-reaction assay of a clinical sample may be useful to provide a more rapid result than culture, but the assay is less sensitive, especially when performed on blood samples. ${ }^{6}$ Serologic testing alone is not adequate to confirm the diagnosis, especially in endemic regions where background seropositivity is common.? The treatment of melioidosis includes prolonged courses of appropriate antibiotics due to the recalcitrant nature of the infection. An initial intensive phase should include at least 10 to 14 days of intravenous ceftazidime or meropenem followed by oral eradication therapy, which comprises trimethoprim-sulfamethoxazole (TMP-SMX) taken every 12 hours for 3 to 6 months, with or without doxycyline. ${ }^{5}$ TMP-SMX plus doxycycline is considered the standard oral eradication regimen, although one recent study showed that TMP-SMX alone is not inferior to TMP-SMX with doxycycline. ${ }^{8}$ Amoxicillin-clavulanate can be used as an alternative agent for eradication therapy when there are contraindications for the use of TMP-SMX. ${ }^{9}$

Mortality rates in melioidosis vary from region to region. For instance, it is approximately $40 \%$ in northeast Thailand, ${ }^{5}$ but $14 \%$ in Australia. ${ }^{2}$ Recurrent melioidosis occurs in approximately 1 in 16 patients, often in the first year after the initial presentation. Nearly $25 \%$ of recurrences are due to reinfection, with the remainder due to relapses from a persistent focus of infection. ${ }^{10}$

In conclusion, septic arthritis only occurs in $4 \%$ of patients presenting with melioidosis. ${ }^{2}$ When there is diffuse pulmonary involvement, it may mimic disseminated tuberculosis, other acute disseminated or focal sepsis syndromes, and systemic vasculitis syndromes. ${ }^{1,2}$ This case is relevant to the medical literature as melioidosis is becoming an emerging infection and expanding its territories worldwide. It should be considered early in the differential diagnoses of patients presenting with constitutional symptoms in areas where it is known to be endemic so that treatment can be started early to reduce its high mortality and morbidity. ${ }^{2,3}$ 


\section{References}

1. Wiersinga WJ, Currie BJ, Peacock SJ. Melioidosis. N Engl J Med. 2012 Sep 13;367(11):1035-44.

2. Currie BJ, Ward L, Cheng AC. The epidemiology and clinical spectrum of melioidosis: 540 cases from the 20 year Darwin prospective study. PLoS Negl Trop Dis. 2010 Nov 30;4(11):e900.

3. Limmathurotsakul D, Wongratanacheewin $S$, Teerawattanasook N, Wongsuvan $\mathrm{G}$, Chaisuksant $\mathrm{S}$, Chetchotisakd $\mathrm{P}$, et al. Increasing incidence of human melioidosis in Northeast Thailand. Am J Trop Med Hyg. 2010 Jun;82(6):1113-7. 4. Pagalavan L. Melioidosis: the Johor Bahru Experience. Med J Malaysia. 2005 Dec;60(5):599-605.

5. Peacock SJ, Schweizer HP, Dance DA, Smith TL, Gee JE, Wuthiekanun V, et al. Management of accidental laboratory exposure to Burkholderia pseudomallei and B. mallei. Emerg Infect Dis. 2008 Jul;14(7):e2.

6. Richardson LJ, Kaestli M, Mayo M, Bowers JR, Tuanyok A, Schupp J, et al. Towards a rapid molecular diagnostic for melioidosis: comparison of DNA extraction methods from clinical specimens. J Microbiol Methods. 2012 Jan;88(1):179-81.
7. Wuthiekanun V, Chierakul W, Langa S, Chaowagul W, Panpitpat C, Saipan $P$, et al. Development of antibodies to Burkholderia pseudomallei during childhood in melioidosis-endemic northeast Thailand. Am J Trop Med Hyg. 2006 Jun; 74(6):1074-5.

8. Chetchotisakd P, Chierakul W, Chaowagul W, Anunnatsiri S, Phimda K, Mootsikapun P, et al. Trimethoprim-sulfamethoxazole versus trimethoprim-sulfamethoxazole plus doxycycline as oral eradicative treatment for melioidosis (MERTH): a multicentre, double-blind, non-inferiority, randomised controlled trial. Lancet. 2014 Mar 1;383(9919);807-14.

9. Cheng AC, Chierakul W, Chaowagul W, Chetchotisakd P, Limmathurotsakul D, Dance DA, et al. Consensus guidelines for dosing of amoxicillin-clavulanate in melioidosis. Am J Trop Med Hyg. 2008 Feb;78(2):208-9.

10. Limmathurotsakul D, Chaowagul W, Chierakul W, Stepniewska K, Maharjan $B$, Wuthiekanun $\mathrm{V}$, et al. Risk factors for recurrent melioidosis in northeast Thailand. Clin Infect Dis. 2006 Oct 15;43(8):979-86.

\section{Acknowledgments}

We would like to thank the Department of Microbiology and Department of Radiology of Segamat Hospital for their assistance.

\section{Conflict of Interest Statement a Funding}

The author has no funding, financial relationships or conflicts of interest to disclose.

\section{Author Contributions}

Conception and design the work/idea: HSL, AZAR. Collect data/obtaining results, Analysis and interpretation of data: HSL, SHY. Write the manuscript: HSL. Critical revision of the manuscript, Approval of the final version: AZAR.

Cite as:

Lee HS, Ahamed-Riyaaz AA, Yeoh SH. Acute Disseminated Melioidosis Presenting with Septic Arthritis and Diffuse Pulmonary Consolidation in an Otherwise Healthy Adult: A Case Report. Int J Med Students. 2014 Nov-2015 Mar;3(1):59-62. 